\title{
The index and spectrum of Lie poset algebras of types B, C, and D
}

\author{
Vincent E. Coll, Jr. \\ Department of Mathematics \\ Lehigh University \\ Bethlehem, U.S.A. \\ vec208@lehigh.edu
}

\author{
Nicholas W. Mayers \\ Department of Mathematics \\ Lehigh University \\ Bethlehem, U.S.A. \\ nwm5095@gmail.com
}

\author{
Nicholas Russoniello \\ Department of Mathematics \\ Lehigh University \\ Bethlehem, U.S.A. \\ nvr217@lehigh.com
}

Submitted: May 2, 2020; Accepted: Aug 3, 2021; Published: Aug 27, 2021

(C) The authors. Released under the CC BY license (International 4.0).

\begin{abstract}
We define posets of types B, C, and D. These posets encode the matrix forms of certain Lie algebras which lie between the algebras of upper-triangular and diagonal matrices. Our primary concern is the index and spectral theories of such typeB, C, and D Lie poset algebras. For an important restricted class, we develop combinatorial index formulas and, in particular, characterize posets corresponding to Frobenius Lie algebras. In this latter case we show that the spectrum is binary; that is, consists of an equal number of 0's and 1's. Interestingly, type-B, C, and D Lie poset algebras can be related to Reiner's notion of a parset.
\end{abstract}

Mathematics Subject Classifications: 17B99, 05E15

\section{Introduction}

The study of "Lie poset algebras" was initiated by Coll and Gerstenhaber in [2], where the deformation theory of such algebras was investigated. The authors define Lie poset algebras as subalgebras of $A_{n-1}=\mathfrak{s l}(n)$ which lie between the subalgebras of uppertriangular and diagonal matrices; we will refer to such Lie subalgebras of $\mathfrak{s l}(n)$ as type- $A$ Lie poset algebras. In [2], the authors suggest a way in which to extend the notion of Lie 
poset algebra to the other classical families of Lie algebras. Interestingly, the resulting Lie algebras can be related to the notion of a "parset" as defined by Reiner (see Remark 6 and cf. $[\mathbf{2 2}, \mathbf{2 3}]$ ). Following the suggestion of [2], we define posets ${ }^{1}$ which encode the matrix forms of such Lie poset algebras and, here, initiate an investigation into their index and spectral theories.

Formally, the index of a Lie algebra $\mathfrak{g}$ is defined as

$$
\text { ind } \mathfrak{g}=\min _{F \in \mathfrak{g}^{*}} \operatorname{dim}\left(\operatorname{ker}\left(B_{F}\right)\right) \text {, }
$$

where $B_{F}$ is the skew-symmetric Kirillov form defined by $B_{F}(x, y)=F([x, y])$, for all $x, y \in \mathfrak{g}$. Of particular interest are those Lie algebras which have index zero, and are called Frobenius. ${ }^{2}$ A functional $F \in \mathfrak{g}^{*}$ for which $\operatorname{dim}\left(\operatorname{ker}\left(B_{F}\right)\right)=$ ind $\mathfrak{g}=0$ is likewise called Frobenius. Given a Frobenius Lie algebra $\mathfrak{g}$ and a Frobenius functional $F \in \mathfrak{g}^{*}$, the map $\mathfrak{g} \rightarrow \mathfrak{g}^{*}$ defined by $x \mapsto B_{F}(x,-)$ is an isomorphism. The inverse image of $F$ under this isomorphism, denoted $\widehat{F}$, is called a principal element of $\mathfrak{g}$ (see $[\mathbf{1 0}, \mathbf{1 6}]$ ). In [18], Ooms shows that the eigenvalues (and multiplicities) of $\operatorname{ad}(\widehat{F})=[\widehat{F},-]: \mathfrak{g} \rightarrow \mathfrak{g}$ do not depend on the choice of principal element $\widehat{F}$. It follows that the spectrum of $\operatorname{ad}(\widehat{F})$ is an invariant of $\mathfrak{g}$, which we call the spectrum of $\mathfrak{g}$ (see $[\mathbf{1}, \mathbf{3}, \mathbf{7}]$ ).

The development of combinatorial index formulas for certain families of Lie algebras is of current interest. Families for which such formulas have been found include seaweed algebras and type-A Lie poset algebras (see $[4,5,6,8,9,12,17,19,20,21]$ ). In this article, we consider the analogues of type-A Lie poset algebras in the other classical types: $B_{k}=\mathfrak{s o}(2 k+1), C_{k}=\mathfrak{s p}(2 k)$, and $D_{k}=\mathfrak{s o}(2 k)$; such algebras are called type- $B, C$, and $D$ Lie poset algebras, respectively. We find that these Lie poset algebras are encoded by certain posets whose underlying sets are of the form $\{-n, \ldots,-1,0,1, \ldots, n\}$ in type $\mathrm{B}$ and of the form $\{-n, \ldots,-1,1, \ldots, n\}$ in types $\mathrm{C}$ and D. Furthermore, we fully develop the index and spectral theories of type-B, C, and D Lie poset algebras whose underlying posets have the property that there are no relations between pairs of positive integers and no relations between pairs of negative integers. In particular, for this important base case, we develop combinatorial index formulas which rely on an associated graph - called a relation graph (see Theorem 44). These formulas allow us to fully characterize Frobenius, type-B, C, and D Lie poset algebras in this case (see Theorem 49). Finally, using an isomorphism result (see Theorem 56), we are able to show that Frobenius, type-B, C, and D Lie poset algebras encoded by posets whose chains are of cardinality at most two have a binary spectrum; that is, a spectrum consisting of an equal number of 0's and 1's (see Theorem 60).

The organization of this paper is as follows. Section 2 sets the combinatorial definitions and notation needed from the theory of posets. In Sections 3 and 4 we formally introduce

\footnotetext{
${ }^{1}$ Such posets are similar in structure to those attached to the signed posets (type-B parsets) of Reiner by Fischer in his Thesis (see Remark 11 and [13]).

${ }^{2}$ Frobenius algebras are of special interest in deformation and quantum group theory stemming from their connection with the classical Yang-Baxter equation (see [14, 15]).
} 
type-B, C, and D Lie poset algebras and the posets which encode them. Sections 5 and 6 deal with the index and spectral theories of type-B, C, and D Lie poset algebras. Finally, in a short epilogue, we compare the less complicated type-A case with the type- $\mathrm{B}, \mathrm{C}$, and D cases developed here.

\section{Posets}

A finite poset $\left(\mathcal{P}, \preceq_{\mathcal{P}}\right)$ consists of a finite set $\mathcal{P}$ together with a binary relation $\preceq_{\mathcal{P}}$ on $\mathcal{P}$ which is reflexive, anti-symmetric, and transitive. When no confusion will arise, we simply denote a poset $\left(\mathcal{P}, \preceq_{\mathcal{P}}\right)$ by $\mathcal{P}$, and $\preceq_{\mathcal{P}}$ by $\preceq$. Throughout, we let $\leqslant$ denote the natural ordering on $\mathbb{Z}$. Two posets $\mathcal{P}$ and $\mathcal{Q}$ are isomorphic if there exists an order-preserving bijection $\mathcal{P} \rightarrow \mathcal{Q}$.

Remark 1. If $|\mathcal{P}|=n$, then there exists a poset $\left(\{1, \ldots, n\}, \leqslant^{\prime}\right)$ and $f:\left(\mathcal{P}, \preceq_{\mathcal{P}}\right) \rightarrow$ $\left(\{1, \ldots, n\}, \leqslant^{\prime}\right)$ such that $\leqslant^{\prime} \subseteq \leqslant$ and $f$ is an isomorphism. Thus, given a poset $\left(\mathcal{P}, \preceq_{\mathcal{P}}\right)$ with $|\mathcal{P}|=n$, unless stated otherwise, we assume that $\mathcal{P}=\{1, \ldots, n\}$ and $\preceq_{\mathcal{P}} \subseteq \leqslant$.

Let $x, y \in \mathcal{P}$. If $x \preceq y$ and $x \neq y$, then we call $x \preceq y$ a strict relation and write $x \prec y$. If $x \prec y$ and there exists no $z \in \mathcal{P}$ satisfying $x \prec z \prec y$, then $y$ covers $x$ and $x \prec y$ is a covering relation. Covering relations are used to define a visual representation of $\mathcal{P}$ called the Hasse diagram - a graph whose vertices correspond to elements of $\mathcal{P}$ and whose edges correspond to covering relations (see, for example, Figure 1).

Example 2. Let $\mathcal{P}=\{1,2,3,4\}$ with $1 \prec 2 \prec 3,4$. In Figure 1 we illustrate the Hasse diagram of $\mathcal{P}$.

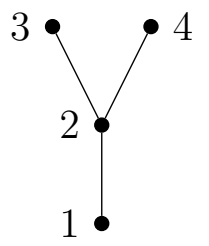

Figure 1: Hasse diagram of a poset

Given a subset $S \subset \mathcal{P}$, the induced subposet generated by $S$ is the poset $\mathcal{P}_{S}$ on $S$, where, for $x, y \in S, x \prec_{\mathcal{P}_{S}} y$ if and only if $x \prec_{\mathcal{P}} y$. A totally ordered subset $S \subset \mathcal{P}$ is called a chain. Finally, we define the dual of $\mathcal{P}$, denoted $\mathcal{P}^{*}$, to be the poset on the same set as $\mathcal{P}$ with $j \prec_{\mathcal{P}^{*}} i$ if and only $i \prec_{\mathcal{P}} j$.

\section{Lie poset algebras}

Let $\mathcal{P}$ be a finite poset and $\mathbf{k}$ be an algebraically closed field of characteristic zero, which we may take to be the complex numbers. The (associative) incidence algebra $A(\mathcal{P})=A(\mathcal{P}, \mathbf{k})$ 
is the span over $\mathbf{k}$ of elements $e_{i, j}$, for $i, j \in \mathcal{P}$ satisfying $i \preceq j$, with product given by setting $e_{i, j} e_{k, l}=e_{i, l}$ if $j=k$ and 0 otherwise. The trace of an element $\sum c_{i, j} e_{i, j}$ is $\sum c_{i, i}$.

We can equip $A(\mathcal{P})$ with the commutator product $[a, b]=a b-b a$, where juxtaposition denotes the product in $A(\mathcal{P})$, to produce the Lie poset algebra $\mathfrak{g}(\mathcal{P})=\mathfrak{g}(\mathcal{P}, \mathbf{k})$. If $|\mathcal{P}|=n$, then both $A(\mathcal{P})$ and $\mathfrak{g}(\mathcal{P})$ may be regarded as subalgebras of the algebra of $n \times n$ uppertriangular matrices over $\mathbf{k}$. Such a matrix representation is realized by replacing each basis element $e_{i, j}$ by the $n \times n$ matrix $E_{i, j}$ containing a 1 in the $i, j$-entry and 0's elsewhere. The (associative) product between elements $e_{i, j}$ is then replaced by matrix multiplication between the $E_{i, j}$.

Example 3. Let $\mathcal{P}$ be the poset of Example 2. The matrix form of elements in $\mathfrak{g}(\mathcal{P})$ is illustrated in Figure 2, where the *'s denote potential non-zero entries from $\mathbf{k}$.

1
2
3
4 $\left[\begin{array}{llll}1 & 2 & 3 & 4 \\ * & * & * & * \\ 0 & * & * & * \\ 0 & 0 & * & 0 \\ 0 & 0 & 0 & *\end{array}\right]$

Figure 2: Matrix form of $\mathfrak{g}(\mathcal{P})$, for $\mathcal{P}=\{1,2,3,4\}$ with $1 \prec 2 \prec 3,4$

Remark 4. Restricting $\mathfrak{g}(\mathcal{P})$ to trace-zero matrices results in a subalgebra of $A_{n-1}=\mathfrak{s l}(n)$, which we call a type-A Lie poset algebra (see $[\mathbf{2}, \mathbf{6}, \mathbf{7}]$ ). In [2], the authors show that type-A Lie poset algebras are equivalent to subalgebras of $\mathfrak{s l}(n)$ lying between the Borel subalgebra of $\mathfrak{s l}(n)$ consisting of all upper-triangular matrices and its Cartan subalgebra of diagonal matrices. Coll and Mayers [6, 7] initiated an investigation into the index and spectral theories of type-A Lie poset algebras.

In light of Remark 4, we make the following definition.

Definition 5. Let $\mathfrak{g}$ be one of the classical simple Lie algebras. Let $\mathfrak{h} \subset \mathfrak{g}$ be the Cartan subalgebra consisting of all diagonal matrices and $\mathfrak{b} \subset \mathfrak{g}$ be its associated Borel subalgebra consisting of all upper-triangular matrices. A Lie subalgebra $\mathfrak{p} \subset \mathfrak{g}$ satisfying $\mathfrak{h} \subseteq \mathfrak{p} \subseteq \mathfrak{b}$ is called a Lie poset subalgebra of $\mathfrak{g}$. If $\mathfrak{g}$ is $A_{n-1}=\mathfrak{s l}(n), B_{n}=\mathfrak{s o}(2 n+1), C_{n}=\mathfrak{s p}(2 n)$, or $D_{n}=\mathfrak{s o}(2 n)$, for $n \in \mathbb{Z}_{>0}$, then $\mathfrak{p}$ is called a type-A, type-B, type-C, or type-D Lie poset algebra, respectively.

Remark 6 . Let $\mathcal{P}$ be a poset with underlying set $\{1, \ldots, n\}$ which does not necessarily satisfy $\preceq_{\mathcal{P}} \subseteq \leqslant$. In [22], Reiner describes a method for identifying $\mathcal{P}$ with a subset of the root system corresponding to $\mathfrak{s l}(n)$. Reiner then generalizes this construction to produce what he calls a "parset." If $\Phi$ is a root system, then a parset (partial root system) is defined to be a subset $P \subset \Phi$ such that

- $\alpha \in P$ implies $-\alpha \notin P$; and 
- if $\alpha_{1}, \alpha_{2} \in P$ and $c_{1} \alpha_{1}+c_{2} \alpha_{2} \in \Phi$ for some $c_{1}, c_{2}>0$, then $c_{1} \alpha_{1}+c_{2} \alpha_{2} \in P$.

Lie poset algebras of classical type correspond to a subclass of parsets in the following way. If $\mathfrak{h}$ is a Cartan subalgebra of a simple Lie algebra $\mathfrak{g}$, then choose a Borel subalgebra $\mathfrak{b}$. Let $\Phi^{+}$be the set of positive roots in $\Phi$, and let $P \subset \Phi^{+}$satisfy if $\alpha_{1}, \alpha_{2} \in P$ and $c_{1} \alpha_{1}+c_{2} \alpha_{2} \in \Phi^{+}$for some $c_{1}, c_{2}>0$, then $c_{1} \alpha_{1}+c_{2} \alpha_{2} \in P$. If $\mathfrak{n}_{P}=\bigoplus_{\alpha \in P} g_{\alpha}$, where $\mathfrak{g}_{\alpha}$ is the root space of $\mathfrak{g}$ corresponding to $\alpha$, then a Lie poset algebra of classical type is conjugate to the algebra $\mathfrak{h} \oplus \mathfrak{n}_{P}$.

\section{Posets of types B, C, and D}

In this section, we provide definitions for posets of types $\mathrm{B}, \mathrm{C}$, and $\mathrm{D}$ (cf. [6]). These posets encode matrix forms that define Lie poset algebras of types B, C, and D.

Remark 7. Recall that the subalgebra of upper-triangular matrices of

- $\mathfrak{s p}(2 n)$ consists of $2 n \times 2 n$ matrices of the form given in Figure 3 with $N=\widehat{N}$,

- $\mathfrak{s o}(2 n)$ consists of $2 n \times 2 n$ matrices of the form given in Figure 3 with $N=-\widehat{N}$, and

- $\mathfrak{s o}(2 n+1)$ consists of $2 n+1 \times 2 n+1$ matrices of the form given in Figure 3 with $N=-\widehat{N}$ and a 0 on the diagonal separating $M$ and $-\widehat{M}$,

where $\widehat{N}$ denotes the transpose of $N$ with respect to the antidiagonal.

$$
\left(\begin{array}{cc}
M & N \\
0 & -\widehat{M}
\end{array}\right)
$$

Figure 3: Matrix form

Remark 8. Throughout the remainder of this article, unless stated otherwise, we assume that the rows and columns of a $2 n \times 2 n$ (resp., $2 n+1 \times 2 n+1$ ) matrix are labeled by $\{-n, \ldots,-1,1, \ldots, n\}$ (resp., $\{-n, \ldots,-1,0,1, \ldots, n\}$ ).

Theorem 9. For $n \in \mathbb{Z}_{>0}$, set $[n]=\{1, \ldots, n\}$. A basis for $a$

- type-C Lie poset algebra can be taken which consists solely of elements of the form $E_{-i,-i}-E_{i, i}$, for all $i \in[n], E_{-i,-j}-E_{j, i}$, for $i \neq j \in[n], E_{-i, j}+E_{-j, i}$, for $i \neq j \in[n]$, and $E_{-i, i}$, for $i \in[n]$.

- type-D Lie poset algebra can be taken which consists solely of elements of the form $E_{-i,-i}-E_{i, i}$, for all $i \in[n], E_{-i,-j}-E_{j, i}$, for $i \neq j \in[n]$, and $E_{-i, j}-E_{-j, i}$, for $i \neq j \in[n]$. 
- type-B Lie poset algebra can be taken which consists solely of elements of the form $E_{-i,-i}-E_{i, i}$, for all $i \in[n], E_{-i,-j}-E_{j, i}$, for $i \neq j \in[n], E_{-i, j}-E_{-j, i}$, for $i \neq j \in[n]$, and $E_{-j, 0}-E_{0, j}$, for $j \in[n]$.

Proof. We prove the result for type-C Lie poset algebras as the type-B and D cases follow similarly. Let $\mathfrak{g} \subset \mathfrak{s} \mathfrak{p}(2 n)$ be a type-C Lie poset algebra and

$$
\mathscr{B}_{n}=\left\{E_{-i,-j}-E_{j, i} \mid i \neq j \in[n]\right\} \cup\left\{E_{-i, j}+E_{-j, i} \mid i \neq j \in[n]\right\} \cup\left\{E_{-i, i} \mid i \in[n]\right\} .
$$

We claim that if $x \in \mathscr{B}_{n}$ occurs as a summand with nonzero coefficient in an element of $\mathfrak{g}$, then $x \in \mathfrak{g}$. Since $\mathfrak{g}$ is a type-C Lie poset algebra, $\mathfrak{g}$ contains the Cartan subalgebra $\mathfrak{h} \subset \mathfrak{s p}(2 n)$ of diagonal matrices; that is, $E_{-i,-i}-E_{i, i} \in \mathfrak{g}$, for all $i \in[n]$. Thus, letting $\mathfrak{n} \subset$ $\mathfrak{s p}(2 n)$ denote the subalgebra of strictly upper-triangular matrices, it is sufficient to show that if $a=\sum_{k=1}^{r} c_{k} x_{k} \in \mathfrak{g} \cap \mathfrak{n}$, where $x_{k} \in \mathscr{B}_{n}$, for $k \in[r]$, then $x_{k} \in \mathfrak{g} \cap \mathfrak{n}$, for some $k \in[r]$. If not, suppose that the given $a \in \mathfrak{g}$ has minimal $r$ such that no summand $x_{k}$ is contained in $\mathfrak{g} \cap \mathfrak{n}$; surely, $r \geqslant 2$. If there exists $x_{k}$, a summand of $a$, such that $x_{k}=E_{-i, i}$, for $i \in[n]$, set $d=E_{-i,-i}-E_{i, i}$; otherwise, there exists $x_{k}$ such that $x_{k}=E_{-j,-i}-E_{i, j}$ or $E_{-j, i}+E_{-i, j}$, in which case set $d=E_{i, i}-E_{-i,-i}+E_{-j,-j}-E_{j, j}$ or $d=E_{-i,-i}-E_{i, i}+E_{-j,-j}-E_{j, j}$, respectively. In either case, $[d, a]$ is not a multiple of $a$ but is a linear combination of the same summands $x_{k}$, for $k \in[r]$. To see this, note that $\left[d, x_{k}\right]=2 x_{k}$, while $\left[d, x_{l}\right]=d_{l} x_{l}$ with $d_{l}=-1,0,1$, for $l \neq k$. Thus, there is a linear combination of $a$ and $[d, a]$ which is nonzero and contains no more than $r-1$ of the summands $x_{k}$, for $k \in[r]$; one of them is consequently already in $\mathfrak{g} \cap \mathfrak{n}$, a contradiction. Thus, since $\left\{E_{-i,-i}-E_{i, i} \mid i \in[n]\right\} \cup \mathscr{B}_{n}$ forms a basis of $\mathfrak{s p}(2 n)$, the set $\left\{E_{-i,-i}-E_{i, i} \mid i \in[n]\right\}$ can be extended to a basis of $\mathfrak{g}$ with the desired form.

Definition 10. A type- $\mathrm{C}$ poset is a poset $\mathcal{P}=\{-n, \ldots,-1,1, \ldots, n\}$ such that

1. if $i \prec_{\mathcal{P}} j$, then $i<j$; and

2. if $i \neq-j$, then $i \prec_{\mathcal{P}} j$ if and only if $-j \prec_{\mathcal{P}}-i$.

A type- $\mathrm{D}$ poset is a poset $\mathcal{P}=\{-n, \ldots,-1,1, \ldots, n\}$ satisfying 1 and 2 above as well as

3. $i$ does not cover $-i$, for $i \in\{1, \ldots, n\}$.

A type-B poset is a poset $\mathcal{P}=\{-n, \ldots,-1,0,1, \ldots, n\}$ satisfying 1 through 3 above.

Remark 11. In his thesis [13], Fischer attaches posets to the signed posets (type-B parsets) of Reiner in order to study their homology. Given the close relationship between parsets and Lie poset algebras, it should come as no surprise that the two classes of associated posets overlap.

Example 12. In Figure 4, we illustrate the Hasse diagram of the type-C (and D) poset $\mathcal{P}=\{-3,-2,-1,1,2,3\}$ with $-2 \prec 1,3$ and $-3,-1 \prec 2$. Note that adding 0 to $\mathcal{P}$ and a vertex labeled 0 to the Hasse diagram of Figure 4 results in a type-B poset and its corresponding Hasse diagram. 


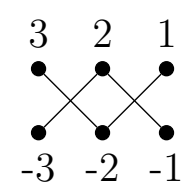

Figure 4: Hasse diagram of a type-C poset

Theorem 13. Type- $C$ (resp., $B$ or $D$ ) posets $\mathcal{P}$ are in bijective correspondence with type-C (resp., $B$ or $D)$ Lie poset algebras $\mathfrak{p}$ as follows:

- $-i, i \in \mathcal{P}$ if and only if $E_{-i,-i}-E_{i, i} \in \mathfrak{p}$;

- $-i \prec_{\mathcal{P}}-j$ and $j \prec_{\mathcal{P}} i$ if and only if $E_{-i,-j}-E_{j, i} \in \mathfrak{p}$, for $i \neq j$;

- $-i \prec_{\mathcal{P}} j$ and $-j \prec_{\mathcal{P}} i$ if and only if $E_{-i, j}+E_{-j, i} \in \mathfrak{p}\left(\right.$ resp., $\left.E_{-i, j}-E_{-j, i} \in \mathfrak{p}\right)$, for $i \neq j$;

and only in type- $C$,

- $-i \prec_{\mathcal{P}} i$ if and only if $E_{-i, i} \in \mathfrak{p}$.

Proof. We prove the result for type-C posets and type-C Lie poset algebras. The proofs for the types-B and $\mathrm{D}$ cases are similar, only requiring minor modifications. Let $\mathcal{P}$ be a type-C poset. The collection of matrices generated by the elements in $\mathfrak{p}$ clearly consists of upper-triangular matrices in $\mathfrak{s p}(|\mathcal{P}|)$ and includes the Cartan of diagonal matrices. Furthermore, transitivity in $\mathcal{P}$ guarantees closure of $\mathfrak{p}$ under the Lie bracket. Thus, $\mathfrak{p}$ is a type-C Lie poset algebra.

Conversely, if $\mathfrak{p}$ is a type-C Lie poset algebra, then taking the basis guaranteed by Theorem 9, we may use the correspondence outlined in the statement of the current theorem to form a set $\mathcal{P}$ together with a relation $\preceq_{\mathcal{P}}$ between its elements. The relations generated by the given basis elements clearly force $\preceq_{\mathcal{P}}$ to satisfy all properties required of a type-C poset except transitivity - but transitivity is equivalent to the closure of $\mathfrak{p}$ under the Lie bracket. The result follows.

Remark 14. Note that as in the type-A case, type-C posets $\mathcal{P}$ determine the matrix form of the corresponding type-C Lie poset algebra by identifying which entries of a $|\mathcal{P}| \times|\mathcal{P}|$ matrix can be non-zero. In particular, the $i, j$-entry can be non-zero if and only if $i{ }_{\mathcal{P}} j$. The same is almost true in types- $\mathrm{B}$ and $\mathrm{D}$, except one ignores relations of the form $-i \prec_{\mathcal{P}} i$.

Example 15. Let $\mathcal{P}$ be the poset of Example 12. The matrix form encoded by $\mathcal{P}$ and defining the corresponding type-C (and D) Lie poset algebra is illustrated in Figure 5, where $*$ 's denote potential non-zero entries from $\mathbf{k}$. 


-3
-2
-1
1
2
3 $\left[\begin{array}{cccccc}* & -2 & -1 & 1 & 2 & 3 \\ 0 & * & 0 & 0 & * & 0 \\ 0 & 0 & * & 0 & * & 0 \\ 0 & 0 & 0 & * & 0 & 0 \\ 0 & 0 & 0 & 0 & * & 0 \\ 0 & 0 & 0 & 0 & 0 & *\end{array}\right]$

Figure 5: Matrix form for $\mathcal{P}=\{-3,-2,-1,1,2,3\}$ with $-2 \prec 1,3$ and $-3,-1 \prec 2$

Remark 16. Given a type-C poset $\mathcal{P}$, we denote the corresponding type-C Lie poset algebra by $\mathfrak{g}_{C}(\mathcal{P})$; furthermore, we define the following basis for $\mathfrak{g}_{C}(\mathcal{P})$ :

$$
\begin{aligned}
\mathscr{B}_{C}(\mathcal{P})=\left\{E_{-i,-i}-E_{i, i} \mid \pm i \in \mathcal{P}\right\} & \cup\left\{E_{-i,-j}-E_{j, i} \mid \pm i, \pm j \in \mathcal{P},-i \prec-j, j \prec i\right\} \\
& \cup\left\{E_{-i, j}+E_{-j, i} \mid \pm i, \pm j \in \mathcal{P},-j \prec i,-i \prec j\right\} \\
& \cup\left\{E_{-i, i} \mid \pm i \in \mathcal{P},-i \prec i\right\} .
\end{aligned}
$$

Similarly, given a type-D poset $\mathcal{P}$, we denote the corresponding type-D Lie poset algebra by $\mathfrak{g}_{D}(\mathcal{P})$ and define a basis as follows:

$$
\begin{aligned}
\mathscr{B}_{D}(\mathcal{P})=\left\{E_{-i,-i}-E_{i, i} \mid \pm i \in \mathcal{P}\right\} & \cup\left\{E_{-i,-j}-E_{j, i} \mid \pm i, \pm j \in \mathcal{P},-i \prec-j, j \prec i\right\} \\
& \cup\left\{E_{-i, j}-E_{-j, i} \mid \pm i, \pm j \in \mathcal{P},-j \prec i,-i \prec j, j<i\right\} .
\end{aligned}
$$

The definitions of $\mathfrak{g}_{B}(\mathcal{P})$ and $\mathscr{B}_{B}(\mathcal{P})$ carry over with the obvious modifications from the type-D analogues.

Theorem 17. If $\mathcal{P}$ is a type-D poset such that $-i \nprec i$, for all $i \in \mathcal{P}$, then $\mathfrak{g}_{D}(\mathcal{P})$ is isomorphic to $\mathfrak{g}_{C}(\mathcal{P})$.

Proof. The result follows by comparing the structure constants of $\mathfrak{g}_{D}(\mathcal{P})$ and $\mathfrak{g}_{C}(\mathcal{P})$ corresponding to the bases $\mathscr{B}_{D}(\mathcal{P})$ and $\mathscr{B}_{C}(\mathcal{P})$, respectively.

Theorem 18. If $\mathcal{P}$ is a type- $B$ poset for which 0 is not related to any other element of $\mathcal{P},-i \nprec i$, for all $i \in \mathcal{P}$, and $\mathcal{P}_{0}=\mathcal{P}_{\mathcal{P} \backslash\{0\}}$, then $\mathfrak{g}_{B}(\mathcal{P})$ is isomorphic to $\mathfrak{g}_{C}\left(\mathcal{P}_{0}\right)$.

Proof. In this case $\mathscr{B}_{D}\left(\mathcal{P}_{0}\right)$ forms a basis for both $\mathfrak{g}_{B}(\mathcal{P})$ and $\mathfrak{g}_{D}\left(\mathcal{P}_{0}\right)$. Applying Theorem 17 establishes the result.

We continue to set the combinatorial notation for posets of types B, C, and D.

Given a type-B, C, or D poset $\mathcal{P}$, let $\mathcal{P}^{+}=\mathcal{P}_{\mathcal{P} \cap \mathbb{Z}_{>0}}$ and $\mathcal{P}^{-}=\mathcal{P}_{\mathcal{P} \cap \mathbb{Z}_{<0}}$; that is, $\mathcal{P}^{+}$ (resp., $\mathcal{P}^{-}$) is the poset induced by the positive (resp., negative) elements of $\mathcal{P}$.

Remark 19. By property 2 of Definition 10, we have that $\mathcal{P}^{+}$is isomorphic to $\left(\mathcal{P}^{-}\right)^{*}$.

Let $\operatorname{Re}_{ \pm}(\mathcal{P})$ denote the set of relations $x \prec_{\mathcal{P}} y$ such that $x \in \mathcal{P}^{-}$and $y \in \mathcal{P}^{+}$. We call $\mathcal{P}$ separable if $\operatorname{Rel}_{ \pm}(\mathcal{P})=\varnothing$, and non-separable otherwise. We say that $\mathcal{P}$ is of height $(i, j)$ if $i$ (resp., $j$ ) is one less than the largest cardinality of a chain in $\mathcal{P}^{+}$(resp., $\mathcal{P}$ ). 
Example 20. If $\mathcal{P}$ is the poset of Example 12, then $\mathcal{P}^{+}=\{1,2,3\}$ and $\mathcal{P}^{-}=\{-1,-2,-3\}$ where both induced posets have no relations. Further, since $\mathcal{P}$ has chains of cardinality at most one, it is of height $(0,1)$.

To end this section, we introduce a condensed graphical representation for height- $(0,1)$, type-B, C, or D posets which will be used in the following section.

Definition 21. Given a height- $(0,1)$, type-B, C, or D poset $\mathcal{P}$, we define the relation graph $R G(\mathcal{P})$ as follows:

- each pair of elements $-i, i \in \mathcal{P}$ are represented by a single vertex in $R G(\mathcal{P})$ labeled by $i \in \mathcal{P}^{+}$(omitting the vertex representing 0 in type $\mathrm{B}$ );

- if $-i \prec j$ in $\mathcal{P}$, then there is an edge connecting vertex $i$ and vertex $j$ in $R G(\mathcal{P})$.

If $R G(\mathcal{P})$ is connected, then $\mathcal{P}$ is called connected.

Remark 22. Note that $R G(\mathcal{P})$ is well-defined for type-B posets $\mathcal{P}$ since if $\mathcal{P}$ is of height$(0,1)$, then 0 cannot be related to any other element of $\mathcal{P}$. Further, such relation graphs are equivalent to "signed digraphs," as defined by Reiner (see $[22,23]$ ), with the signs removed.

Remark 23. If $-i \prec i$ in $\mathcal{P}$, then vertex $i$ defines a self-loop in $R G(\mathcal{P})$. Note that $R G(\mathcal{P})$ can only contain self-loops if $\mathcal{P}$ is a type-C poset.

Remark 24. Note that normally a poset is called connected if its corresponding Hasse diagram is connected. For the purposes of this paper, though, the notion of connected given in Definition 21 for type-B, C, and D posets is more useful (see Theorem 46).

Example 25. In Figure 6, we illustrate the (a) Hasse diagram and (b) relation graph corresponding to the height- $(0,1)$, type-C poset $\mathcal{P}=\{-3,-2,-1,1,2,3\}$ with $-2 \prec 1,2,3$ and $-3,-1 \prec 2$.

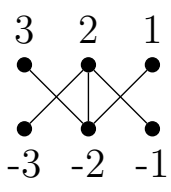

(a)

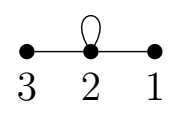

(b)

Figure 6: (a) Hasse diagram and (b) relation graph of a type-C poset

\section{Index}

In this section, we develop combinatorial formulas for the index of type-B, C, and D Lie poset algebras corresponding to height- $(0,1)$, type- $\mathrm{B}, \mathrm{C}$, and D posets, respectively.

It will be convenient to use an alternative characterization of the index. Let $\mathfrak{g}$ be an arbitrary Lie algebra with basis $\left\{x_{1}, \ldots, x_{n}\right\}$. The index of $\mathfrak{g}$ can be expressed using the commutator matrix, $\left(\left[x_{i}, x_{j}\right]\right)_{1 \leqslant i, j \leqslant n}$, over the quotient field $R(\mathfrak{g})$ of the symmetric algebra $\operatorname{Sym}(\mathfrak{g})$ as follows (see [11]). 
Theorem 26. The index of $\mathfrak{g}$ is given by

$$
\text { ind } \mathfrak{g}=n-\operatorname{rank}_{R(\mathfrak{g})}\left(\left[x_{i}, x_{j}\right]\right)_{1 \leqslant i, j \leqslant n} .
$$

Example 27. Consider $\mathfrak{g}_{C}(\mathcal{P})$, for $\mathcal{P}=\{-1,1\}$ with $-1 \prec 1$. A basis for $\mathfrak{g}_{C}(\mathcal{P})$ is given by

$$
\mathscr{B}_{C}(\mathcal{P})=\left\{x_{1}=E_{-1,-1}-E_{1,1}, x_{2}=E_{-1,1}\right\},
$$

where $\left[x_{1}, x_{2}\right]=2 x_{2}$. The commutator matrix $\left(\left[x_{i}, x_{j}\right]\right)_{1 \leqslant i, j \leqslant 2}$ is illustrated in Figure 7 . Since the rank of this matrix is two, it follows from Theorem 26 that $\mathfrak{g}_{C}(\mathcal{P})$ is Frobenius.

$$
\left[\begin{array}{cc}
0 & 2 x_{2} \\
-2 x_{2} & 0
\end{array}\right]
$$

Figure 7: Commutator matrix

Remark 28. To ease notation, row and column labels of commutator matrices will be bolded, and matrix entries will be unbolded. Furthermore, we will refer to the row (resp., column) corresponding to $\mathbf{x}$ in a commutator matrix - and by a slight abuse of notation, in any equivalent matrix - as row (resp., column) $\mathbf{x}$.

Remark 29. For ease of discourse, all results of this section will be stated in terms of typeC Lie poset algebras. Considering Theorems 17 and 18, all results hold in the type-B and D cases as well.

Throughout this section, given a type- $\mathrm{C}$ poset $\mathcal{P}$, we set

$$
\mathcal{C}\left(\mathfrak{g}_{C}(\mathcal{P})\right)=\left(\left[x_{i}, x_{j}\right]\right)_{1 \leqslant i, j \leqslant n} \text {, where }\left\{x_{1}, \ldots, x_{n}\right\}=\mathscr{B}_{C}(\mathcal{P}) .
$$

Theorem 30. If $\mathcal{P}$ is a separable, type- $C$ poset, then

$$
\text { ind } \mathfrak{g}_{C}(\mathcal{P})=\text { ind } \mathfrak{g}\left(\mathcal{P}^{+}\right)=\text {ind } \mathfrak{g}_{A}\left(\mathcal{P}^{+}\right)+1 \text {. }
$$

Proof. Note that

$$
\left\{x_{1}, \ldots, x_{n}\right\}=\left\{E_{i, i} \mid i \in \mathcal{P}^{+}\right\} \cup\left\{E_{i, j} \mid i \prec_{\mathcal{P}^{+}} j\right\}
$$

and

$$
\left\{y_{1}, \ldots, y_{n}\right\}=\left\{\sum_{i=1}^{\left|\mathcal{P}^{+}\right|} E_{i, i}\right\} \cup\left\{E_{i, i}-E_{i+1, i+1}|1 \leqslant i \leqslant| \mathcal{P}^{+} \mid-1\right\} \cup\left\{E_{i, j} \mid i \prec_{\mathcal{P}^{+}} j\right\}
$$

both form bases for $\mathfrak{g}\left(\mathcal{P}^{+}\right)$, while

$$
\left\{z_{1}, \ldots, z_{n-1}\right\}=\left\{E_{i, i}-E_{i+1, i+1}|1 \leqslant i \leqslant| \mathcal{P}^{+} \mid-1\right\} \cup\left\{E_{i, j} \mid i \prec_{\mathcal{P}^{+}} j\right\}
$$

forms a basis for $\mathfrak{g}_{A}\left(\mathcal{P}^{+}\right)$. Thus, $\left(\left[x_{i}, x_{j}\right]\right)_{1 \leqslant i, j \leqslant n}$ and $\left(\left[y_{i}, y_{j}\right]\right)_{1 \leqslant i, j \leqslant n}$ both form commutator matrices of $\mathfrak{g}\left(\mathcal{P}^{+}\right)$, while $\left(\left[z_{i}, z_{j}\right]\right)_{1 \leqslant i, j \leqslant n-1}$ forms a commutator matrix of $\mathfrak{g}_{A}\left(\mathcal{P}^{+}\right)$. Replacing $E_{-i,-i}-E_{i, i}$ by $E_{i, i}$ and $E_{-j,-i}-E_{i, j}$ by $E_{i, j}$ in $\mathcal{C}\left(\mathfrak{g}_{C}(\mathcal{P})\right)$ results in $\left(\left[x_{i}, x_{j}\right]\right)_{1 \leqslant i, j \leqslant n}$, 
establishing the first equality in (1). As for the second equality in (1), since $\sum_{i=1}^{\left|\mathcal{P}^{+}\right|} E_{i, i}$ lies in the center of $\mathfrak{g}\left(\mathcal{P}^{+}\right)$, it follows that

$$
\left(\left[y_{i}, y_{j}\right]\right)_{1 \leqslant i, j \leqslant n}=[0] \oplus\left(\left[z_{i}, z_{j}\right]\right)_{1 \leqslant i, j \leqslant n-1}
$$

establishing the result.

Corollary 31. If $\mathcal{P}$ is a type- $C$ poset such that $\mathfrak{g}_{C}(\mathcal{P})$ is Frobenius, then $\mathcal{P}$ is nonseparable.

Corollary 32. If $\mathcal{P}$ is a height-(0,0), type-C poset, then

$$
\text { ind } \mathfrak{g}_{C}(\mathcal{P})=\left|\mathcal{P}^{+}\right|
$$

Proof. Equation (2) follows since any commutator matrix corresponding to $\mathfrak{g}\left(\mathcal{P}^{+}\right)$is the $\left|\mathcal{P}^{+}\right| \times\left|\mathcal{P}^{+}\right|$zero-matrix.

Remark 33. In light of Corollary 32, the next case to consider is type-C posets $\mathcal{P}$ of height- $(0,1)$. This case is non-trivial and contains the first examples of Frobenius, type-C Lie poset algebras. The remainder of this article concerns the analysis of this case. In particular, using combinatorial index formulas developed in Section 5.2, we are able to fully characterize those height- $(0,1)$, type-C posets which underlie Frobenius, type-C Lie poset algebras (see Theorem 49). A spectral analysis follows in Section 6 .

\subsection{Matrix reduction}

In this section, we describe an algorithm for reducing $\mathcal{C}\left(\mathfrak{g}_{C}(\mathcal{P})\right)$, where $\mathcal{P}$ is a connected, height- $(0,1)$, type-C poset. This reduction facilitates the development of a combinatorial index formula for $\mathfrak{g}_{C}(\mathcal{P})$ in Section 5.2.

As a first step in our matrix reduction, we order the row and column labels of $\mathcal{C}\left(\mathfrak{g}_{C}(\mathcal{P})\right)$, i.e., the elements of $\mathscr{B}_{C}(\mathcal{P})$, as follows:

1. the elements $\mathbf{E}_{-\mathbf{i},-\mathbf{i}}-\mathbf{E}_{\mathbf{i}, \mathbf{i}}$ in increasing order of $i$ in $\mathbb{Z}$;

2. the elements $\mathbf{E}_{-\mathbf{i}, \mathbf{j}}+\mathbf{E}_{-\mathbf{j}, \mathbf{i}}$ in increasing lexicographic order of $(i, j)$, for $i<j$, in $\mathbb{Z} \times \mathbb{Z}$

With this ordering, since height- $(0,1)$, type-C posets have no non-trivial transitivity relations, $\mathcal{C}\left(\mathfrak{g}_{C}(\mathcal{P})\right)$ has the form illustrated in Figure 8.

$$
\left(\begin{array}{cc}
0 & -B(\mathcal{P})^{T} \\
B(\mathcal{P}) & 0
\end{array}\right)
$$

Figure 8: Matrix form of $\mathcal{C}\left(\mathfrak{g}_{C}(\mathcal{P})\right)$, for $\mathcal{P}$ a height- $(0,1)$, type-C poset 
Here, $B(\mathcal{P})$ has rows labeled by basis elements of the form $\mathbf{E}_{-\mathbf{i}, \mathbf{j}}+\mathbf{E}_{-\mathbf{j}, \mathbf{i}}$ and columns labeled by basis elements of the form $\mathbf{E}_{-\mathbf{i},-\mathbf{i}}-\mathbf{E}_{\mathbf{i}, \mathbf{i}}$, and $-B(\mathcal{P})^{T}$ has these labels reversed. Thus, since $\operatorname{rank}(B(\mathcal{P}))=\operatorname{rank}\left(B(\mathcal{P})^{T}\right)$, to calculate the index, it suffices to determine the rank of $B(\mathcal{P})$.

Now, in order to define the matrix reduction, we catalogue the forms of collections of rows in $B(\mathcal{P})$ which correspond to certain substructures of $R G(\mathcal{P})$. Further, we introduce row operations to reduce such collections of rows. To condense illustrations, when no confusion will arise, columns of zeros are omitted.

Paths: If $R G(\mathcal{P})$ has a path consisting of the sequence of vertices $i_{1}, \ldots, i_{n}$, then the corresponding rows and columns of $B(\mathcal{P})$ have the form illustrated in Figure 9.

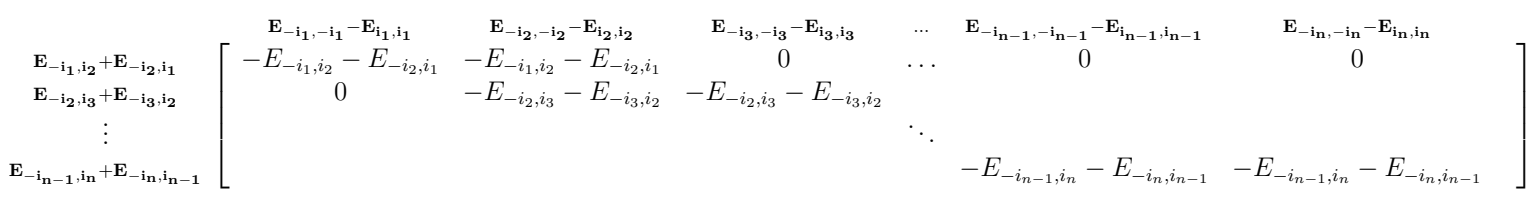

Figure 9: Matrix block corresponding to a path

Definition 34. If $R G(\mathcal{P})$ contains a path consisting of the sequence of vertices $i_{1}, \ldots, i_{n}$, for $n>2$, then define the row operation $\operatorname{Path}\left(i_{1}, \ldots, i_{n}\right)$ on $\mathcal{C}\left(\mathfrak{g}_{C}(\mathcal{P})\right)$ to be

$\left(\mathbf{E}_{-\mathbf{i}_{\mathbf{n}-\mathbf{1}}, \mathbf{i}_{\mathbf{n}}}+\mathbf{E}_{-\mathbf{i}_{\mathbf{n}}, \mathbf{i}_{\mathbf{n}-\mathbf{1}}}\right)+\sum_{j=1}^{n-2}(-1)^{j} \frac{E_{-i_{n-1}, i_{n}}+E_{-i_{n}, i_{n-1}}}{E_{-i_{n-j-1}, i_{n-j}}+E_{-i_{n-j}, i_{n-j-1}}}\left(\mathbf{E}_{-\mathbf{i}_{\mathbf{n}-\mathbf{j}-\mathbf{1}}, \mathbf{i}_{\mathbf{n}-\mathbf{j}}}+\mathbf{E}_{-\mathbf{i}_{\mathbf{n}-\mathbf{j}}, \mathbf{i}_{\mathbf{n}-\mathbf{j}-\mathbf{1}}}\right)$

performed at row $\mathbf{E}_{-\mathbf{i}_{\mathbf{n}-\mathbf{1}}, \mathbf{i}_{\mathbf{n}}}+\mathbf{E}_{-\mathbf{i}_{\mathbf{n}}, \mathbf{i}_{\mathbf{n}-\mathbf{1}}}$.

Example 35. The result of applying $\operatorname{Path}\left(i_{1}, \ldots, i_{n}\right)$ to the matrix of Figure 9 is illustrated in Figure 10.

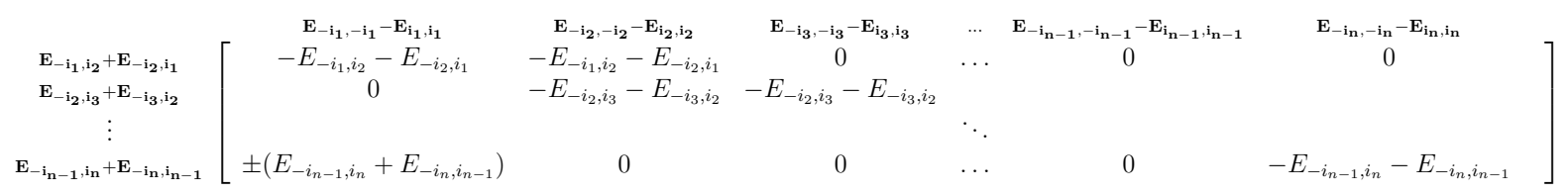

Figure 10: Reduced matrix block corresponding to a path

Self-loop: If $R G(\mathcal{P})$ has a self-loop at vertex $i_{1}$, then the corresponding rows and columns of $B(\mathcal{P})$ have the form illustrated in Figure 11. 


$$
\mathbf{E}_{-\mathbf{i}_{1}, \mathbf{i}_{1}}\left[\begin{array}{c}
\mathbf{E}_{-\mathbf{i}_{1},-\mathbf{i}_{1}}-\mathbf{E}_{\mathbf{i}_{1}, \mathbf{i}_{1}} \\
-2 E_{-i_{1}, i_{1}}
\end{array}\right]
$$

Figure 11: Matrix block corresponding to a self-loop

Ongoing, a self-loop will be considered a cycle consisting of one vertex.

Cycles: If $R G(\mathcal{P})$ contains a cycle consisting of $n>1$ vertices $i_{1}, \ldots, i_{n}$, then the corresponding rows and columns of $B(\mathcal{P})$ have the form illustrated in Figure 12.



Figure 12: Matrix block corresponding to a cycle

Definition 36. If $R G(\mathcal{P})$ contains a cycle consisting of $n>1$ vertices $i_{1}, \ldots, i_{n}$, then define the row operation $\operatorname{Row}_{e}\left(i_{1}, \ldots, i_{n}\right)$ on $\mathcal{C}\left(\mathfrak{g}_{C}(\mathcal{P})\right)$ to be

$$
\left(\mathbf{E}_{-\mathbf{i}_{\mathbf{1}}, \mathbf{i}_{\mathbf{n}}}+\mathbf{E}_{-\mathbf{i}_{\mathbf{n}}, \mathbf{i}_{\mathbf{1}}}\right)+\sum_{j=1}^{n-1}(-1)^{j} \frac{E_{-i_{1}, i_{n}}+E_{-i_{n}, i_{1}}}{E_{-i_{j}, i_{j+1}}+E_{-i_{j+1}, i_{j}}}\left(\mathbf{E}_{-\mathbf{i}_{\mathbf{j}}, \mathbf{i}_{\mathbf{j}+\mathbf{1}}}+\mathbf{E}_{-\mathbf{i}_{\mathbf{j}+\mathbf{1}}, \mathbf{i}_{\mathbf{j}}}\right)
$$

performed at row $\mathbf{E}_{-\mathbf{i}_{1}, \mathbf{i}_{\mathbf{n}}}+\mathbf{E}_{-\mathbf{i}_{\mathbf{n}}, \mathbf{i}_{\mathbf{1}}}$.

Example 37. The result of applying $\operatorname{Row}_{e}\left(i_{1}, \ldots, i_{n}\right)$ to the matrix of Figure 12, for $n$ even, is illustrated in Figure 13.

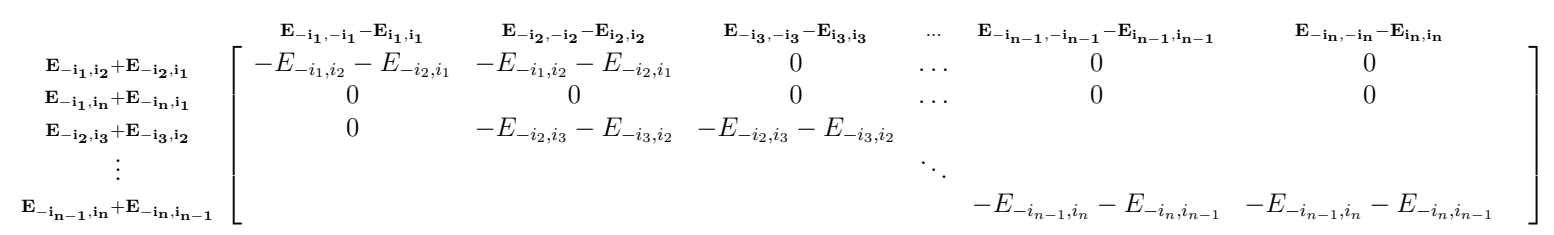

Figure 13: Reduced matrix block corresponding to an even cycle

Remark 38. Note that if we disregard the newly formed zero row in Figure 13, then the configuration of rows is the same as that corresponding to the cycle defined by $i_{1}, \ldots, i_{n}$ with the edge between $i_{1}$ and $i_{n}$ removed.

Definition 39. If $R G(\mathcal{P})$ contains an odd cycle consisting of $n>1$ vertices $i_{1}, \ldots, i_{n}$, then define the row operation $\operatorname{Row}_{o}\left(i_{1}, \ldots, i_{n}\right)$ on $\mathcal{C}\left(\mathfrak{g}_{C}(\mathcal{P})\right)$ to be $\operatorname{Row}_{e}\left(i_{1}, \ldots, i_{n}\right)$ followed by multiplying row $\left(\mathbf{E}_{-\mathbf{i}_{\mathbf{1}}, \mathbf{i}_{\mathbf{n}}}+\mathbf{E}_{-\mathbf{i}_{\mathbf{n}}, \mathbf{i}_{1}}\right)$ by $\frac{E_{-i_{n}, i_{n}}}{E_{-i_{1}, i_{n}}+E_{-i_{n}, i_{1}}}$.

Example 40. The result of applying $\operatorname{Row}_{o}\left(i_{1}, \ldots, i_{n}\right)$ to the matrix of Figure 12 , for $n$ odd, is illustrated in Figure 14. 


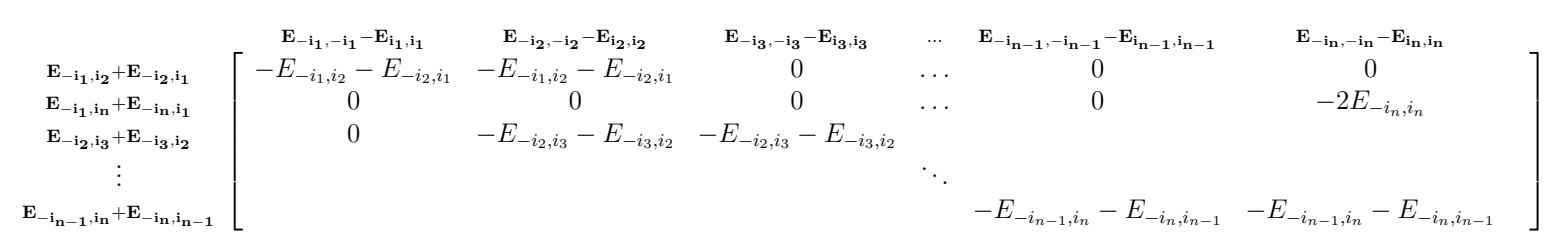

Figure 14: Reduced matrix block corresponding to an odd cycle

Remark 41. Note that the configuration of rows in Figure 14 is the same as that corresponding to the cycle defined by $i_{1}, \ldots, i_{n}$ with the edge between $i_{1}$ and $i_{n}$ removed and with $i_{n}$ defining a self-loop. If vertex $i_{n}$ already defined a self-loop, then row $\mathbf{E}_{-\mathbf{i}_{\mathbf{1}}, \mathbf{i}_{\mathbf{n}}}+\mathbf{E}_{-\mathbf{i}_{\mathbf{n}}, \mathbf{i}_{\mathbf{1}}}$ can be reduced to a zero row in the obvious way.

Now, we come to the matrix reduction algorithm, where the relation graph $R G(\mathcal{P})$ of a connected, height- $(0,1)$, type- $C$ poset $\mathcal{P}$ is used as a bookkeeping device to guide the reduction. If $R G(\mathcal{P})$ contains an odd cycle, then the row-reduction strategy applied in the algorithm uses induction on the number of non-loop edges in $R G(\mathcal{P})$, removing edges from non-loop cycles and removing edges incident to loop vertices. Otherwise, the algorithm uses induction on one less than the number of non-loop edges, removing edges from cycles and then performing reductions for all but one edge in the remaining spanning subtree. At halt, the remaining non-zero rows are clearly linearly independent.

Matrix Reduction Algorithm: Let $\mathcal{P}$ be a connected, height- $(0,1)$, type-C poset

Step 1: Set $G_{1}=R G(\mathcal{P}), M_{1}=B(\mathcal{P}), \Gamma_{1}=\left(G_{1}, M_{1}\right)$, and $k=1$.

Step 2: Check $G_{k}$ for self-loops.

- If $G_{k}$ has a self-loop at vertex $i$ and vertex $i$ is adjacent to a vertex $j$, go to Step 3.

- If $G_{k}$ contains self-loops and no vertex defining a self-loop is adjacent to any other vertex, halt.

- If $G_{k}$ has no self-loops, go to Step 4.

Step 3: Set $l=k$. Form $\Gamma_{l+1}=\left(G_{l+1}, M_{l+1}\right)$ as follows:

1. Perform

$$
\left(\mathbf{E}_{-\mathbf{i}, \mathbf{j}}+\mathbf{E}_{-\mathbf{j}, \mathbf{i}}\right)-\frac{E_{-i, j}+E_{-j, i}}{2 E_{-i, i}} \mathbf{E}_{-\mathbf{i}, \mathbf{i}}
$$

at row $\mathbf{E}_{-\mathbf{i}, \mathbf{j}}+\mathbf{E}_{-\mathbf{j}, \mathbf{i}}$ in $M_{l}$.

2. - If vertex $j$ does not define a self-loop, then:

3. Multiply row $\mathbf{E}_{-\mathbf{i}, \mathbf{j}}+\mathbf{E}_{-\mathbf{j}, \mathbf{i}}$ by $\frac{2 E_{-j, j}}{E_{-i, j}+E_{-j, i}}$ in $M_{l}$.

4. Replace the row label $\mathbf{E}_{-\mathbf{i}, \mathbf{j}}+\mathbf{E}_{-\mathbf{j}, \mathbf{i}}$ by $\mathbf{E}_{-\mathbf{j}, \mathbf{j}}$ in $M_{l}$. 
5. Remove the edge between nodes $i$ and $j$ in $G_{k}$ and add a self-loop at vertex $j$.

6. Set $k=l+1$ and go to Step 2 .

- If vertex $j$ defines a self-loop, then:

3. Perform

$$
\left(\mathbf{E}_{-\mathbf{i}, \mathbf{j}}+\mathbf{E}_{-\mathbf{j}, \mathbf{i}}\right)-\frac{E_{-i, j}+E_{-j, i}}{2 E_{-j, j}} \mathbf{E}_{-\mathbf{j}, \mathbf{j}}
$$

at row $\mathbf{E}_{-\mathbf{i}, \mathbf{j}}+\mathbf{E}_{-\mathbf{j}, \mathbf{i}}$ in $M_{l}$.

4. Replace the row label $\mathbf{E}_{-\mathbf{i}, \mathbf{j}}+\mathbf{E}_{-\mathbf{j}, \mathbf{i}}$ by $\mathbf{0}$ in $M_{l}$.

5. Remove the edge between vertices $i$ and $j$ in $G_{l}$.

6. Set $k=l+1$ and go to Step 2 .

Step 4: Check $G_{k}$ for odd cycles containing $n>1$ vertices

- If $G_{k}$ has such an odd cycle: Let $i_{1}, \ldots, i_{n}$ be the vertices of a largest odd cycle in $G_{k}$; if there are more than one, assuming $i_{1}<i_{j}$ for $j=2, \ldots, n$, take $\left(i_{1}, \ldots, i_{n}\right)$ to be the lexicographically least in $\mathbb{Z}^{n}$. Go to Step 5 .

- If $G_{k}$ has no such odd cycles go to Step 6.

Step 5: Set $l=k$. Form $\Gamma_{l+1}=\left(G_{l+1}, M_{l+1}\right)$ as follows

1. Perform $\operatorname{Row}_{o}\left(i_{1}, \ldots, i_{n}\right)$ in $M_{l}$.

2. Replace the row label $\mathbf{E}_{-\mathbf{i}_{1}, \mathbf{i}_{\mathbf{n}}}+\mathbf{E}_{-\mathbf{i}_{\mathbf{n}}, \mathbf{i}_{\mathbf{1}}}$ by $\mathbf{E}_{-\mathbf{i}_{\mathbf{n}}, \mathbf{i}_{\mathbf{n}}}$ in $M_{l}$.

3. Remove the edge between vertices $i_{1}$ and $i_{n}$, and add a self-loop at vertex $i_{n}$ in $G_{l}$.

4. Set $k=l+1$ and go to Step 2

Step 6: Check $G_{k}$ for even cycles:

- If $G_{k}$ has an even cycle: Let $i_{1}, \ldots, i_{n}$ be the vertices of a largest even cycle in $G_{k}$; if there are more than one, assuming $i_{1}<i_{j}$ for $j=2, \ldots, n$, take $\left(i_{1}, \ldots, i_{n}\right)$ to be the lexicographically least in $\mathbb{Z}^{n}$. Go to Step 7 .

- If $G_{k}$ has no even cycles, go to Step 8 .

Step 7: Set $l=k$. Form $\Gamma_{l+1}=\left(G_{l+1}, M_{l+1}\right)$ as follows

1. Perform $\operatorname{Row}_{e}\left(i_{1}, \ldots, i_{n}\right)$ in $M_{l}$.

2. Replace the row label $\mathbf{E}_{-\mathbf{i}_{1}, \mathbf{i}_{\mathbf{n}}}+\mathbf{E}_{-\mathbf{i}_{\mathbf{n}}, \mathbf{i}_{\mathbf{1}}}$ by $\mathbf{0}$ in $M_{l}$.

3. Remove the edge between vertices $i_{1}$ and $i_{n}$ in $G_{l}$. 
4. Set $k=l+1$ and go to Step 2 .

Step 8: Set $l=k$. Form $\Gamma_{l+1}=\left(G_{l+1}, M_{l+1}\right)$ as follows

1. Take $i_{0}$ minimal in $\mathbb{Z}$ such that $i_{0}$ is a degree one vertex of $G_{l}$ and assume that all other vertices of $G_{l}$ are of maximal distance $n$ from $i_{0}$.

2. Working from $m=n$ down to 2 , for all paths of length $m$ in $G_{l}$ starting at $i_{0}$, say $i_{0}, \ldots, i_{m}$, perform $\operatorname{Path}\left(i_{0}, \ldots, i_{m}\right)$ in $M_{l}$.

3. Halt.

Remark 42. Since we only consider finite posets $\mathcal{P}$, the above algorithm must halt in a finite number of steps.

\subsection{Index formula}

In this section, we determine an index formula for type-C Lie poset algebras corresponding to height- $(0,1)$, type-C posets. Throughout, let $V(\mathcal{P})$ and $E(\mathcal{P})$ denote, respectively, the set of vertices and edges of $R G(\mathcal{P})$.

Lemma 43. Let $\mathcal{P}$ be a connected, height- $(0,1)$, type- $C$ poset.

- If $R G(\mathcal{P})$ contains an odd cycle, then the rank of $B(\mathcal{P})$ in $\mathcal{C}\left(\mathfrak{g}_{C}(\mathcal{P})\right)$ is $|V(\mathcal{P})|$.

- If $R G(\mathcal{P})$ contains no odd cycles, then the rank of $B(\mathcal{P})$ in $\mathcal{C}\left(\mathfrak{g}_{C}(\mathcal{P})\right)$ is $|V(\mathcal{P})|-1$.

Proof. The proof breaks into 4 cases.

Case 1: If $R G(\mathcal{P})$ contains a self-loop, then the algorithm proceeds by removing adjacent edges to vertices defining self-loops and making sure, post edge removal, that such adjacent vertices define self-loops. Thus, as $R G(\mathcal{P})$ is assumed to be connected, this implies that the algorithm above halts with $\Gamma_{n}=\left(G_{n}, M_{n}\right)$, where

- $G_{n}=R G\left(\mathcal{P}^{\prime}\right)$ for the poset $\mathcal{P}^{\prime}$ satisfying $\mathcal{P}^{\prime}=\mathcal{P}$ as sets and $-i \prec \mathcal{P}^{\prime} i$, for all $i \in \mathcal{P}^{\prime}$, and

- $M_{n}$ is the $B\left(\mathcal{P}^{\prime}\right)$ block in $\mathcal{C}\left(\mathfrak{g}_{C}\left(\mathcal{P}^{\prime}\right)\right)$ with (potentially) additional zero rows;

that is, each of the $\left|\mathcal{P}^{+}\right|=|V(\mathcal{P})|$ columns of $M_{n}$ has a corresponding unique row with unique nonzero entry in that column. Thus, the result follows.

Case 2: If $R G(\mathcal{P})$ contains an odd cycle consisting of $n>1$ vertices and no self-loops, then the algorithm starts by removing an edge from an odd cycle and adding a self-loop. The corresponding matrix $M_{k}$ reflects these changes. From here, the algorithm proceeds, and the result follows, as in Case 1. 
Case 3: If $R G(\mathcal{P})$ is a tree, then the algorithm halts at $\Gamma_{n}=\left(G_{n}, M_{n}\right)$, where if $i_{0}$ is the specified degree one vertex of Step 8, then given $i_{j}$ and $i_{k}$ adjacent in $R G(\mathcal{P})$ with $i_{j}$ contained in the unique path from $i_{0}$ to $i_{k}$ we have that row $\mathbf{E}_{-\mathbf{i}_{\mathbf{j}}, \mathbf{i}_{\mathbf{k}}}-\mathbf{E}_{-\mathbf{i}_{\mathbf{k}}, \mathbf{i}_{\mathbf{j}}}$ is the unique row with nonzero entry in column $\mathbf{E}_{-\mathbf{i}_{\mathbf{k}},-\mathbf{i}_{\mathbf{k}}}-\mathbf{E}_{\mathbf{i}_{\mathbf{k}}, \mathbf{i}_{\mathbf{k}}}$; that is, all $\left|\mathcal{P}^{+}\right|-1=|V(\mathcal{P})|-1$ rows of $M_{n}$ are linearly independent and the result follows.

Case 4: If $R G(\mathcal{P})$ contains an even cycle and no odd cycles, then the algorithm removes edges from even cycles (introducing zero rows), until the resulting graph is a tree. The corresponding matrix $M_{k}$ reflects these changes. From here, the algorithm proceeds, and the result follows, as in Case 3.

As a result of Lemma 43, we get the following.

Theorem 44. If $\mathcal{P}$ is a connected, height- $(0,1)$, type- $C$ poset, then

$$
\text { ind } \mathfrak{g}_{C}(\mathcal{P})=|E(\mathcal{P})|-|V(\mathcal{P})|+2 \delta_{\circ},
$$

where $\delta_{\circ}$ is the indicator function for $R G(\mathcal{P})$ containing no odd cycles.

Proof. To start, by Theorem 26, we know that

$$
\text { ind } \mathfrak{g}_{C}(\mathcal{P})=\operatorname{dim}\left(\mathcal{C}\left(\mathfrak{g}_{C}(\mathcal{P})\right)\right)-\operatorname{rank}\left(\mathcal{C}\left(\mathfrak{g}_{C}(\mathcal{P})\right)\right) \text {, }
$$

where $\operatorname{dim}\left(\mathcal{C}\left(\mathfrak{g}_{C}(\mathcal{P})\right)\right)=|E(\mathcal{P})|+|V(\mathcal{P})|$. Furthermore, $\operatorname{rank}\left(\mathcal{C}\left(\mathfrak{g}_{C}(\mathcal{P})\right)\right)=2 \cdot \operatorname{rank}(B(\mathcal{P}))$. By Lemma 43, we know that if $R G(\mathcal{P})$ contains an odd cycle, then $\operatorname{rank}(B(\mathcal{P}))=|V(\mathcal{P})|$; that is,

$$
\text { ind } \mathfrak{g}_{C}(\mathcal{P})=|E(\mathcal{P})|+|V(\mathcal{P})|-2|V(\mathcal{P})|=|E(\mathcal{P})|-|V(\mathcal{P})| \text {. }
$$

Otherwise, $\operatorname{rank}(B(\mathcal{P}))=|V(\mathcal{P})|-1$ so that

$$
\text { ind } \mathfrak{g}_{C}(\mathcal{P})=|E(\mathcal{P})|+|V(\mathcal{P})|-2(|V(\mathcal{P})|-1)=|E(\mathcal{P})|-|V(\mathcal{P})|+2 \text {. }
$$

The result follows.

Remark 45. Note that if $R G(\mathcal{P})$ is not connected, then the elements of $\mathcal{P}$ corresponding to each connected component $K_{i}$ of $R G(\mathcal{P})$ induce posets $\mathcal{P}_{K_{i}}$ which are isomorphic to connected, type-C posets of height- $(0,0)$ or $(0,1)$.

Theorem 46. If $\mathcal{P}$ is a height- $(0,1)$, type-C poset such that $R G(\mathcal{P})$ consists of connected components $\left\{K_{1}, \ldots, K_{n}\right\}$, then

$$
\text { ind } \mathfrak{g}_{C}(\mathcal{P})=\sum_{i=1}^{n} \text { ind } \mathfrak{g}_{C}\left(\mathcal{P}_{K_{i}}\right) \text {. }
$$

Proof. Note that basis elements of $\mathfrak{g}_{C}(\mathcal{P})$ corresponding to different connected components of $R G(\mathcal{P})$ have trivial bracket relations. Thus, $\mathcal{C}\left(\mathfrak{g}_{C}(\mathcal{P})\right)$ can be arranged to be block diagonal with each block corresponding to the basis elements of a connected component of $R G(\mathcal{P})$. Since the block corresponding to $K_{i}$ is equal, after potentially relabelling elements of $\mathcal{P}_{K_{i}}$, to $\mathcal{C}\left(\mathfrak{g}_{C}\left(\mathcal{P}_{K_{i}}\right)\right)$, for $1 \leqslant i \leqslant n$, the result follows. 
Combining Theorems 44 and 46 with Corollary 32 we get the following.

Theorem 47. If $\mathcal{P}$ is a height- $(0,1)$, type- $C$ poset, then

$$
\text { ind } \mathfrak{g}_{C}(\mathcal{P})=|E(\mathcal{P})|-|V(\mathcal{P})|+2 \eta(\mathcal{P}),
$$

where $\eta(\mathcal{P})$ denotes the number of connected components of $R G(\mathcal{P})$ containing no odd cycles.

Remark 48. To ease discourse in what follows, type-C (resp., B or D) posets corresponding to Frobenius, type-C (resp., B or D) Lie poset algebras are referred to as Frobenius, type-C (resp., B or D) posets.

Theorem 49. If $\mathcal{P}$ is a height- $(0,1)$, type-C poset, then $\mathfrak{g}_{C}(\mathcal{P})$ is Frobenius if and only if each connected component of $R G(\mathcal{P})$ contains a single cycle which consists of an odd number of vertices.

Proof. Let $\mathcal{P}$ be a a height- $(0,1)$, type-C poset. Combining Theorem 46 and Corollary 32 , we find that $\mathcal{P}$ is Frobenius if and only if $\mathcal{P}$ is a disjoint sum of Frobenius, height- $(0,1)$, type-C posets. Assume $\mathcal{P}$ is connected. Note that $|E(\mathcal{P})|-|V(\mathcal{P})| \geqslant-1$ with equality when $R G(\mathcal{P})$ is a tree. Thus, by Theorem 44, if $\mathcal{P}$ is Frobenius, then $R G(\mathcal{P})$ must contain an odd cycle. If $R G(\mathcal{P})$ contains an odd cycle, then $|E(\mathcal{P})|-|V(\mathcal{P})| \geqslant 0$ with equality if and only if $R G(\mathcal{P})$ contains a single odd cycle. Therefore, the result follows.

\section{Spectrum}

In this section, we show that Frobenius, height- $(0,1)$ type- $\mathrm{B}, \mathrm{C}$, or $\mathrm{D}$ posets encode Lie poset algebras whose spectrum is binary; that is, consists of an equal number of 0's and 1 's. To accomplish this, we establish an isomorphism result concerning a more general class of Frobenius Lie algebras. The aforementioned isomorphism result allows us to focus our attention on the spectrum of a family of Lie algebras with simple bracket relations. By combining the isomorphism and spectrum results, we are able to further conclude that Frobenius, height- $(1,1)$ type-B, C, or D posets encode Lie poset algebras whose spectrum is also binary.

Before proceeding to the isomorphism result, we first generalize Definition 5 of Section 3.

Definition 50. Given a semisimple Lie algebra $\mathfrak{g}$, let $\mathfrak{h} \subset \mathfrak{g}$ be a Cartan subalgebra, and let $\mathfrak{b} \subset \mathfrak{g}$ be an associated Borel subalgebra. A Lie subalgebra $\mathfrak{p} \subset \mathfrak{g}$ satisfying $\mathfrak{h} \subseteq \mathfrak{p} \subseteq \mathfrak{b}$ is called a Lie poset subalgebra.

Remark 51. Throughout this section, unless stated otherwise, the term Lie poset algebra will refer to a Lie poset subalgebra of a semisimple Lie algebra.

Remark 52. Note that Definition 5 is recovered from Definition 50 by fixing $\mathfrak{g}$ to be one of the classical families of Lie algebras, and fixing $\mathfrak{b}$ and $\mathfrak{h}$ to be the corresponding Borel subalgebra of upper-triangular matrices and Cartan subalgebra of diagonal matrices, respectively. 
Since Lie poset algebras are evidently solvable, we recall the following basic definition, as we require certain associated terminology for results that follow.

Definition 53. Let $\mathfrak{g}$ be a finite-dimensional Lie algebra. The derived series $\mathfrak{g}=\mathfrak{g}^{0} \supset$ $\mathfrak{g}^{1} \supset \mathfrak{g}^{2} \supset \ldots$ is defined by $\mathfrak{g}^{i}=\left[\mathfrak{g}^{i-1}, \mathfrak{g}^{i-1}\right]$, for $i>0$. The least $k$ for which $\mathfrak{g}^{k} \neq 0$ and $\mathfrak{g}^{j}=0$, for $j>k$, is called the derived length of $\mathfrak{g}$. The Lie algebra $\mathfrak{g}$ is solvable if its derived series terminates in the the zero subalgebra. If a Lie algebra has finite derived length $k$, then it is $(k+1)$-step solvable.

Remark 54 . Note that a height- $(0,1)$ or $(1,1)$ type-B, C, or D poset encodes a two-step solvable type-B, C, or D Lie poset algebra, respectively.

We require the following lemma, which generalizes Theorem 9, to show that a Lie poset algebra inherits a Cartan-Weyl basis from its parent semisimple Lie algebra. Recall that a Cartan-Weyl basis of a Lie algebra, $\mathfrak{g}$, is defined to be a basis consisting of generators of the Cartan subalgebra, $\mathfrak{h} \subset \mathfrak{g}$, along with root vectors, $E_{\alpha}$, where $\alpha \in \Phi$ - the root system of $\mathfrak{g}$. The multiplication of Cartan-Weyl basis vectors takes the form $\left[h_{i}, h_{j}\right]=0$ for all $i, j \in\{1, \ldots, \operatorname{dim} \mathfrak{h}\}$, and $\left[h_{i}, E_{\alpha}\right]=\alpha\left(h_{i}\right) E_{\alpha}$. In fact, the lemma goes further to guarantee that any subalgebra containing the Cartan of the parent semisimple Lie algebra has such a basis.

Lemma 55. Let $\mathfrak{g}$ be a semisimple Lie algebra with Cartan subalgebra $\mathfrak{h}$ and Cartan-Weyl basis $\mathscr{B}(\mathfrak{g})$. All Lie subalgebras of $\mathfrak{g}$ containing $\mathfrak{h}$ have a Cartan-Weyl basis. In particular, if $\mathfrak{g}^{\prime}$ is a Lie subalgebra of $\mathfrak{g}$ and $\mathfrak{h} \subset \mathfrak{g}^{\prime}$, then there exists a basis, $\mathscr{B}\left(\mathfrak{g}^{\prime}\right)$, of $\mathfrak{g}^{\prime}$ such that $\mathscr{B}\left(\mathfrak{g}^{\prime}\right) \subset \mathscr{B}(\mathfrak{g})$.

Proof. Let $\mathscr{B}(\mathfrak{g})=\left\{h_{1}, \ldots, h_{n}, w_{1}, \ldots, w_{m}\right\}$, where $\left\{h_{1}, \ldots, h_{n}\right\}$ forms a basis of $\mathfrak{h}$. Since $\mathfrak{h} \subset \mathfrak{g}^{\prime}$, it suffices to show that if $a=\sum_{i=1}^{r} a_{i} w_{i} \in \mathfrak{g}^{\prime}$, then at least one of the $w_{i}$ is contained in $\mathfrak{g}^{\prime}$; this will imply that all such $w_{i}$ are contained in $\mathfrak{g}^{\prime}$ and we can extend $\left\{h_{1}, \ldots, h_{n}\right\}$ to a basis $\mathscr{B}\left(\mathfrak{g}^{\prime}\right) \subset \mathscr{B}(\mathfrak{g})$. Assume that the given $a \in \mathfrak{g}^{\prime}$ has minimal $r$ such that no summand $w_{i}$ is contained in $\mathfrak{g}^{\prime}$; surely $r \geqslant 2$. If $[h, a]=c_{h} a$, for all $h \in \mathfrak{h}$, then $a$ and its summands, $w_{i}$, are elements of the same root space of $\mathfrak{g}$. Hence, since root spaces of $\mathfrak{g}$ are one-dimensional, $a=c w_{i}$ for some summand, $w_{i}$, of $a$, a contradiction. Thus, there must exist $h \in \mathfrak{h}$ such that $[h, a]$ is not a multiple of $a$ but is a linear combination of the same collection of summands $w_{i}$. This implies that there is a linear combination of $a$ and $[h, a]$ which is not zero and contains no more than $r-1$ of these summands. One of them is consequently already in $\mathfrak{g}^{\prime}$, a contradiction.

Theorem 56. Frobenius, two-step solvable Lie poset algebras are isomorphic if and only if the algebras have the same dimension.

Proof. Let $\mathfrak{g}$ be a Frobenius, two-step solvable Lie poset algebra and let $\mathscr{B}(\mathfrak{g})$ be the Cartan-Weyl basis of $\mathfrak{g}$ guaranteed to exist by Lemma 55. Let $D(\mathfrak{g})=\left\{x_{1}, \ldots, x_{n}\right\} \subset$ $\mathscr{B}(\mathfrak{g})$ consist of the basis elements of $\mathfrak{h} \subset \mathfrak{g}$ and $T(\mathfrak{g})=\mathscr{B}(\mathfrak{g}) \backslash D(\mathfrak{g})=\left\{x_{n+1}, \ldots, x_{n+m}\right\}$. Define the commutator matrix $\mathcal{C}(\mathfrak{g})=\left(\left[x_{i}, x_{j}\right]\right)_{1 \leqslant i, j \leqslant n+m}$, where

$$
\mathscr{B}(\mathfrak{g})=\left\{x_{1}, \ldots, x_{n+m}\right\}
$$


with the elements of $D(\mathfrak{g})$ occurring first, followed by the elements of $T(\mathfrak{g})$. Note that, with the given ordering, $\mathcal{C}(\mathfrak{g})$ has the form illustrated in Figure 15 , where $B(\mathfrak{g})$ has rows labeled by elements of $D(\mathfrak{g})$ and columns labeled by elements of $T(\mathfrak{g})$, and $-B(\mathfrak{g})^{T}$ has these labels reversed.

$$
\left(\begin{array}{cc}
0 & B(\mathfrak{g}) \\
-B(\mathfrak{g})^{T} & 0
\end{array}\right)
$$

Figure 15: Matrix form of $\mathcal{C}(\mathfrak{g})$

To see this, since $[\mathfrak{h}, \mathfrak{h}]=0$, it suffices to show that $[x, y]=0$, for all $x, y \in T(\mathfrak{g})$. Assume for a contradiction that there exists $z \in \mathfrak{g}$ such that $[x, y]=z \neq 0$, for some $x, y \in T(\mathfrak{g})$. Since $x, y \notin D(\mathfrak{g})$, there exist $h_{x}, h_{y} \in \mathfrak{h}$ such that $\left[h_{x}, x\right]=k_{1} x$ and $\left[h_{y}, y\right]=k_{2} y$, for $k_{1}, k_{2} \neq 0$. Thus, $x, y \in \mathfrak{g}^{1}$ and $z \in \mathfrak{g}^{2}$, a contradiction.

Considering Theorem 26, since $\mathfrak{g}$ is Frobenius, $\mathcal{C}(\mathfrak{g})$ has full rank. Consequently, $B(\mathfrak{g})$ also has full rank. Note that this implies $n=m$. Now, since the rows of $B(\mathfrak{g})$ are labeled by elements of $D(\mathfrak{g}) \subset \mathfrak{h}$, the entries in the column labeled by $x \in T(\mathfrak{g})$ are all multiples of $x$; that is, $B(\mathfrak{g})$ has the form illustrated in Figure 16 , where $c_{i, j} \in \mathbf{k}$ for $1 \leqslant i \leqslant n$ and $n+1 \leqslant j \leqslant 2 n$.

$$
B(\mathfrak{g})=\begin{gathered}
x_{1} \\
x_{2} \\
\vdots \\
x_{n}
\end{gathered}\left[\begin{array}{cccc}
x_{n+1} & x_{n+2} & \cdots & x_{2 n} \\
c_{1, n+1} x_{n+1} & c_{1, n+2} x_{n+2} & \cdots & c_{1,2 n} x_{2 n} \\
c_{2, n+1} x_{n+1} & c_{2, n+2} x_{n+2} & \cdots & c_{2,2 n} x_{2 n} \\
\vdots & \vdots & \cdots & \vdots \\
c_{n, n+1} x_{n+1} & c_{n, n+2} x_{n+2} & \cdots & c_{n, 2 n} x_{2 n}
\end{array}\right]=\left[c_{i, n+j}\right]_{1 \leqslant i, j \leqslant n}\left[\begin{array}{cccc}
x_{1, n+1} & 0 & \cdots & 0 \\
0 & x_{2, n+2} & \cdots & 0 \\
\vdots & \vdots & \cdots & \vdots \\
0 & 0 & \cdots & x_{n, 2 n}
\end{array}\right]
$$

Figure 16: Matrix form of $B(\mathfrak{g})$

Since $B(\mathfrak{g})$ has full rank, it follows that the matrix $C=\left[c_{i, n+j}\right]_{1 \leqslant i, j \leqslant n} \in \mathbf{k}^{n \times n}$ is invertible. Applying $C^{-1} \in \mathbf{k}^{n \times n}$ to $\left[x_{1}, x_{2}, \ldots, x_{n}\right]^{T}$ provides a new basis $\left\{d_{1}, d_{2}, \ldots, d_{n}\right\}$ of $\mathfrak{h}$ satisfying $\left[d_{i}, x_{n+j}\right]=\delta_{i, j} x_{n+j}$, for $1 \leqslant i, j \leqslant n$. Thus, setting $e_{i}=x_{n+i}$, there exists a basis $\left\{d_{1}, e_{1}, \ldots, d_{n}, e_{n}\right\}$ for $\mathfrak{g}$ defined by the bracket relations $\left[d_{i}, e_{i}\right]=e_{i}$, for $i=1, \ldots, n$. Denoting the Lie algebra defined by the basis and bracket relations of the preceding sentence by $\Phi_{n}$, we may conclude that every Frobenius, two-step solvable Lie poset algebra of dimension $2 n$ is isomorphic to $\Phi_{n}$. The result follows.

Remark 57. Note that the Lie algebra $\Phi_{n}$ found in the proof of Theorem 56 is just the direct sum of $n$ copies of the unique, 2-dimensional, non-abelian Lie algebra.

Corollary 58. Frobenius, type-B, $C$, and D Lie poset algebras encoded by height- $(0,1)$ or $(1,1)$ type- $B, C$, or $D$ posets, respectively, are isomorphic if and only if the algebras have the same dimension. 
Considering the results above, to show that Frobenius, type-B, C, and D Lie poset algebras encoded by height- $(0,1)$ or $(1,1)$ type-B, C, or D posets, respectively, have a binary spectrum, we need only prove that the Frobenius Lie algebra $\Phi_{n}$ has a binary spectrum.

Theorem 59. $\Phi_{n}$ has a binary spectrum.

Proof. Let $e_{i}^{*}$, for $i=1, \ldots, n$, denote the functional on $\Phi_{n}$ which returns the coefficient of $e_{i}$. A straightforward exercise in linear algebra shows that $f=\sum_{i=1}^{n} e_{i}^{*}$ is a Frobenius functional on $\Phi_{n}$ with principal element $\widehat{f}=\sum_{i=1}^{n} d_{i}$. Calculating $\left[\widehat{f}, d_{i}\right]$ and $\left[\widehat{f}, e_{i}\right]$, for $i=1, \ldots, n$, establishes the result.

We have the following immediate corollary.

Theorem 60. If $\mathfrak{g}$ is a Frobenius, type-B, $C$, or D Lie poset algebra encoded by a height$(0,1)$ or $(1,1)$ type- $B, C$, or $D$ poset, respectively, then $\mathfrak{g}$ has a binary spectrum.

\section{$7 \quad$ Epilogue}

For type-A Lie poset algebras, there are no restrictions on the underlying poset, so the notion of "height" is less complicated. In the type-A setting, the height of a poset $\mathcal{P}$ is defined to be one less than the largest cardinality of a chain. If $\mathcal{P}$ is a connected, height-one (type-A) poset, then the first two authors recently established that the index of the associated type-A Lie poset algebra $\mathfrak{g}_{A}(\mathcal{P})$ is given by the following closed-form expression (see Theorem 4, [6]):

$$
\text { ind } \mathfrak{g}_{A}(\mathcal{P})=|E(\mathcal{P})|-|V(\mathcal{P})|+1
$$

where $E(\mathcal{P})$ and $V(\mathcal{P})$ are, respectively, the sets of edges and vertices of the Hasse diagram of $\mathcal{P}$.

For a connected, height- $(0,1)$ type-C poset $\mathcal{Q}$, the Hasse diagram is replaced by the relations graph $R G(\mathcal{Q})$ and the type-C analogue of $(3)$ is given by Theorem 44:

$$
\text { ind } \mathfrak{g}_{C}(\mathcal{Q})=|E(\mathcal{Q})|-|V(\mathcal{Q})|+2 \delta_{o},
$$

where $E(\mathcal{Q})$ and $V(\mathcal{Q})$ are, respectively, the sets of edges and vertices of $R G(\mathcal{Q})$, and $\delta_{o}$ is the indicator function for the existence of odd cycles in $R G(\mathcal{Q})$. (Of course, equation (4) remains valid with "C" replaced by "B" or " $\mathrm{D}$ ".)

As with Frobenius, type-C Lie poset algebras corresponding to height- $(0,1)$ or $(1,1)$ posets - but more generally - the spectrum of $\mathfrak{g}_{A}(\mathcal{P})$ is binary when $\mathcal{P}$ is of height two or less. In fact, even more is known: if $\mathcal{P}$ is a toral poset (see [7]) of arbitrary height for which $\mathfrak{g}_{A}(\mathcal{P})$ is Frobenius, then the spectrum of $\mathfrak{g}_{A}(\mathcal{P})$ is binary. We conjecture that having a binary spectrum is a property shared by all Frobenius Lie poset algebras in all of the classical types. 


\section{Acknowledgements}

The authors are indebted to an anonymous referee for their careful reading of the original manuscript and for their insightful suggestions which enhanced both the exposition and clarity of several proofs.

\section{References}

[1] A. Cameron, V. Coll, M. Hyatt, and C. Magnant. "The unbroken spectra of Frobenius seaweeds." arXiv:2108.06019, 2021.

[2] V. Coll and M. Gerstenhaber. "Cohomology of Lie semidirect products and poset algebras." J. Lie Theory, 26(1): 79-95, 2016.

[3] V. Coll, M. Hyatt, and C. Magnant. "The unbroken spectrum of type-A Frobenius seaweeds." J. Algebraic Combin., 48(2): 289-305, 2018.

[4] V. Coll, M. Hyatt, and C. Magnant. "Symplectic meanders." Comm. Algebra, 45(11): 4717-4729, 2017.

[5] V. Coll, M. Hyatt, C. Magnant, and H. Wang. "Meander graphs and Frobenius seaweed Lie algebras II." J. Generalized Lie Theory and Applications, 9(1): 1-7, 2015.

[6] V. Coll and N. Mayers. "The index of Lie poset algebras." J. Combin. Theory Ser. A, 177, 2021.

[7] V. Coll and N. Mayers. "Toral posets and the binary spectrum property." J. Algebraic Combin., 1-29, 2021.

[8] V. Coll, N. Mayers, and N. Russoniello. "The index of nilpotent Lie poset algebras." Linear Algebra Appl., 605: 118-129, 2020.

[9] V. Dergachev and A. Kirillov. "Index of Lie algebras of seaweed type." J. Lie Theory, 10(2): 331-343, 2000.

[10] A. Diatta and B. Manga. "On properties of principal elements of Frobenius Lie algebras." J. Lie Theory, 24(3): 849-864, 2014.

[11] J. Dixmier. "Enveloping Algebras." Graduate Studies in Math. vol 2, AMS, 1996.

[12] A. Elashvili. "On the index of parabolic subalgebras of semisimple Lie algebras." Unpublished preprint, 1990.

[13] S. Fischer. "Signed poset homology and q-analog Möbius functions." Doctoral Dissertation, University of Michigan, 1993.

[14] M. Gerstenhaber and A. Giaquinto. "Boundary solutions of the classical Yang-Baxter equation." Letters Math. Physics, 40(4): 337-353, 1997.

[15] M. Gerstenhaber and A. Giaquinto. "Graphs, Frobenius functionals, and the classical Yang-Baxter equation." arXiv:0808.2423, 2008.

[16] M. Gerstenhaber and A. Giaquinto. "The principal element of a Frobenius Lie algebra." Letters Math. Physics, 88: 333-341, 2009. 
[17] A. Joseph. "On semi-invariants and index for biparabolic (seaweed) algebras, I." J. Algebra, 305(1): 487-515, 2006.

[18] A. Ooms. "On Frobenius Lie algebras." Comm. Algebra, 8(1): 13-52, 1980.

[19] D. Panyushev. "Inductive formulas for the index of seaweed Lie algebras." Mosc. Math. J., 1(2): 221-241, 2001.

[20] D. Panyushev and O. Yakimova. "On seaweed subalgebras and meander graphs in type C." Pacific J. Math., 285(2): 485-499, 2016.

[21] D. Panyushev and O. Yakimova. "On seaweed subalgebras and meander graphs in type D." J. Pure Appl. Algebra, 222(11): 3414-3431, 2018.

[22] V. Reiner. "Quotients of Coxeter complexes and P-partitions." vol 460, AMS, 1992.

[23] V. Reiner. "Signed posets." J. Combin. Theory Ser. A, 62(2): 324-360, 1993. 